\title{
Informed consent for biobanking: consensus-based guidelines for adequate comprehension
}

\author{
Laura M. Beskow, MPH, PhD ${ }^{1,2}$, Carrie B. Dombeck, MA ${ }^{1}$, Cole P. Thompson, BA ${ }^{1}$, \\ J. Kemp Watson-Ormond, BA ${ }^{1}$ and Kevin P. Weinfurt, PhD $^{1,3}$
}

Purpose: Federal regulations and best practice guidelines identify categories of information that should be communicated to prospective biobank participants during the informed consent process. However, uncertainty remains about which of this information participants must understand to provide valid consent.

Methods: We conducted a Delphi process to define "adequate comprehension" in the context of biobanking consent. The process involved an iterative series of three online surveys of a diverse panel of 51 experts, including genome scientists, biobank managers, ethics and policy experts, and community and participant representatives. We sought consensus (>70\% agreement) concerning what specific details participants should know about 16 biobank consent topics.
Results: Consensus was achieved for 15 of the 16 consent topics. The exception was the comprehension needed regarding the Genetic Information Nondiscrimination Act.

Conclusion: Our Delphi process was successful in identifying a concise set of key points that prospective participants must grasp to provide valid consent for biobanking. Specifying the level of knowledge sufficient for individuals to make an informed choice provides a basis for improving consent forms and processes, as well as an absolute metric for assessing the effectiveness of other interventions to improve comprehension.

Genet Med advance online publication 21 August 2014

Key Words: biobanking; comprehension; Delphi process; informed consent; research ethics

\section{INTRODUCTION}

Biobanks and biospecimens are critical components of much medical and translational research. ${ }^{1}$ Numerous biobanking efforts are under way around the world, ${ }^{2,3}$ but informed consent for the collection and storage of materials for future research use remains a challenge. This challenge will be magnified if proposed changes to federal regulations governing human subjects research move forward, as these would require consent for research use of biospecimens, regardless of whether they are initially collected for clinical or research purposes. ${ }^{4}$

The goal of informed consent is to enable competent individuals to decide for themselves whether to participate in research. However, obtaining consent is not simply a matter of disclosing information; promoting comprehension is integral to ensuring that the individual's decision is actually "informed." Valid consent can be critical for protecting the rights and welfare of individuals whose biospecimens are used in research-recent headlines provide a stark reminder of what can otherwise happen..$^{5-8}$

For research involving biobanking, government regulations and best practice guidelines identify key categories of information that should be communicated to prospective participants during the consent process. ${ }^{9} 10$ But little attention has been paid to identifying which of this information prospective participants must grasp to demonstrate adequate understanding. Although participants would perhaps ideally understand every detail of the information deemed important for inclusion in a consent form, such comprehensive knowledge is not necessary to give valid consent. ${ }^{11,12}$

As long as the threshold for adequate comprehension remains unspecified, researchers may fail to identify participants whose consent is not valid or, conversely, deny participation to individuals whose understanding is, in fact, adequate. Significantly, uncertainty as to what constitutes adequate comprehension also undermines efforts to evaluate the sufficiency of consent forms and processes and the development and assessment of interventions to improve them. To address this gap, we conducted a Delphi process with a diverse panel of experts to define "adequate comprehension" in the context of informed consent for biobanking.

\section{MATERIALS AND METHODS}

The Delphi method is an iterative process involving a series of surveys (or "rounds") to gather opinions and seek consensus from a panel of experts. ${ }^{13-18}$ The results of one survey are used to construct the next, and panelists are asked to consider their subsequent responses in light of the results of previous rounds. The absence of an obligation to meet in person lessens constraints

${ }^{1}$ Duke Clinical Research Institute, Duke University School of Medicine, Durham, North Carolina, USA; ${ }^{2}$ Department of Medicine, Duke University School of Medicine, Durham, North Carolina, USA; ${ }^{3}$ Department of Psychiatry and Behavioral Sciences, Duke University School of Medicine, Durham, North Carolina, USA. Correspondence: Laura M. Beskow (laura.beskow@duke.edu) 
on group size and composition. Panelists can be allowed substantial time to consider evidence and reflect on their answers, and the anonymous nature of the process ensures that a single influential panelist does not have a disproportionate impact.

\section{Selection of experts}

Our main consideration in selecting the size and composition of our panel was to ensure the results would be regarded by others as reflecting the consensus of experts representing a range of relevant perspectives. To that end, we recruited panelists from existing committees and consortia that play a leading role in biobanking and human research protections in the United States. We identified and invited panelists based on recognized expertise in (i) biobanking operations/research; (ii) ethics/policy in human subjects research; and/or (iii) community/participant perspectives. The latter included members of community advisory boards for major US biobanks, as well as scholars with extensive experience studying community and participant attitudes and opinions. We confirmed prospective panelists' expertise through an "about you" questionnaire. Those who rated themselves less than "somewhat familiar" with informed consent for biobanking were deemed ineligible. We compensated panelists $\$ 200$ per round; those who did not complete a round were not included in subsequent rounds.

\section{Procedures}

We conducted three online surveys to define "adequate comprehension" for biobanking consent. The surveys were structured around model consent language (Supplementary Appendix S1 online) adapted from established sources ${ }^{19-21}$ and purposely contained ample detail to accommodate a range of opinions about comprehension.

Across all surveys, the panelists' task was to review the model language and, for each of the 16 sections, indicate the minimally correct answer if a prospective participant were asked to explain that topic in his own words. Specifically, we asked panelists to identify the answer that reflects the minimum understanding necessary to provide valid consent. We asked them to imagine that, during the consent process, the study coordinator would compare the participant's answer with the panelist's answer. If the participant's answer was missing key elements of information, the coordinator would review that topic with him in an effort to improve understanding. If the person was still unable to provide the minimally correct answer, he would not be allowed to participate.

A detailed description of the content and analysis of each survey is provided in Supplementary Appendix S2 online. In summary, our approach moved from more qualitative elicitations in early rounds to more quantitative elicitations in later rounds. Results from each were provided to panelists and used as the basis for subsequent surveys. The round 3 survey was explicitly designed to assess consensus quantitatively. Panelists were shown the model consent language, together with one statement per section of what prospective participants would need to say if asked to explain that topic.
We constructed this statement to reflect the greatest degree of agreement developed through the prior rounds. Panelists were asked to rate on a four-point scale how much they agreed or disagreed that the statement generally represented adequate comprehension. There are no established criteria for defining consensus in a Delphi process; suggestions in the literature range from 51 to $80 \% .{ }^{17}$ We selected $70 \%$ agreement as our predetermined threshold.

The Duke University Health System Institutional Review Board deemed this study exempt (45 CFR 46.101(b)(2)). The surveys were implemented online between February and October 2013 using Qualtrics software (Provo, UT).

\section{RESULTS}

\section{Panelist characteristics}

We invited 61 individuals to participate on the panel. Of these, three were determined ineligible, five did not respond, one was unable to participate, and one declined. Those enrolled $(n=51)$ represented a wide range of expertise and considerable familiarity with biobanking consent (Table 1). We maintained a high participation rate throughout the study: 47 (92\% of enrolled) completed all three rounds.

\section{Early rounds}

In round 1, we identified a total of 238 substantive elements of information in panelists' qualitative responses regarding which specific details prospective participants must grasp. Of these elements, 84 were mentioned by at least $20 \%$ of panelists, our threshold for inclusion in the next survey. In round 2, of the 84 elements of information we incorporated, $51(61 \%)$ were endorsed by fewer panelists and 33 (39\%) were endorsed by more panelists compared with round 1 . In general, panelists' round 2 responses moved in the direction suggested by our round 1 results, that is, elements less often endorsed in round 1 tended to be endorsed even less often in round 2 and vice versa (Supplementary Appendix S3 online). Table 2 provides additional details regarding both section- and element-level results from these early rounds.

\section{Final round}

In round 3, consensus was achieved on 15 of the 16 consent topics (Table 3). There was near unanimity on six topics, for which more than $90 \%$ of panelists agreed or strongly agreed that the statement given represented adequate comprehension. For five topics, more than $80 \%$ agreed or strongly agreed with the statement provided.

Three topics exceeded our consensus threshold of 70\% agreement but with slightly more variation of opinion (Certificates of Confidentiality, Blood Draw, Access to Biospecimens/Data). For each of these, panelists who disagreed that the statement represented adequate comprehension most often indicated that it reflected less than prospective participants need to know.

One other topic-Risk - met our consensus threshold, achieving $70 \%$ agreement. Most panelists who disagreed indicated that the statement reflected less than prospective participants need to 
Table 1 Characteristics of Delphi panelists

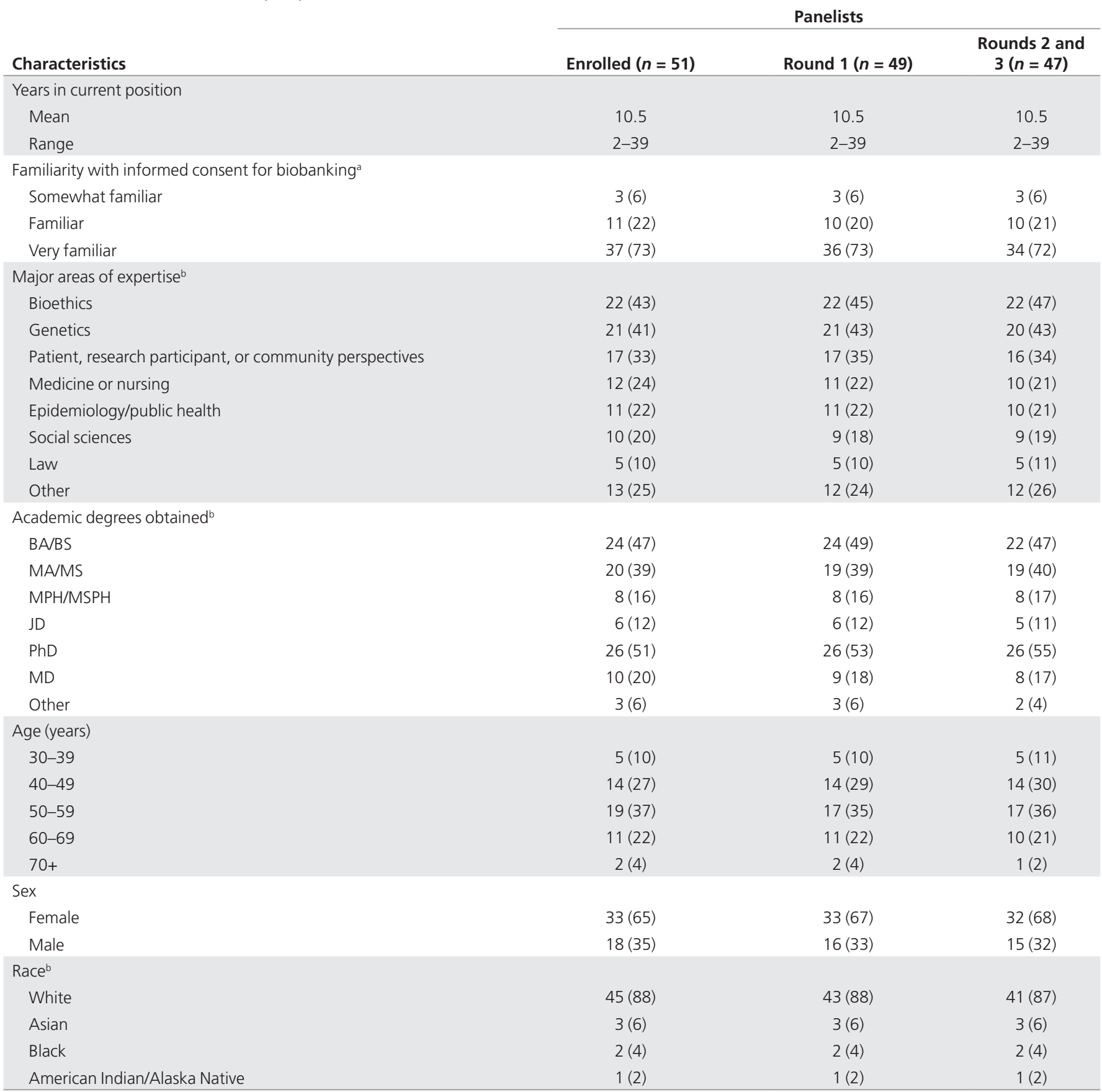

Data are $n(\%)$ unless otherwise indicated. aRespondents who rated themselves as "not too familiar" or "not at all familiar" were deemed ineligible. "Respondents were allowed to choose more than one response.

know, although a nontrivial fraction said it reflected more than required for valid consent.

The topic for which consensus was not achieved was the Genetic Information Nondiscrimination Act (GINA). Results for this topic were disparate in the early rounds, and thus it was the only one for which we presented two separate statements in round 3. Over half (53\%) of panelists agreed with the first option, "There is nothing in this section prospective participants must understand." A slightly larger proportion (60\%) agreed that the second option, "There is a law against discrimination based on my information," represented adequate comprehension, although $26 \%$ felt it reflected less than participants need to know and 15\% felt it reflected more. Panelists' comments at the end of the survey suggested that these differences may be because of skepticism about GINA itself, as well as the opinion that participants should understand the limitations of the law ${ }^{22}$ (Table 4).

Panelists' comments about the Delphi process were substantially positive (Table 4). Many characterized the experience 
Table 2 Summary of early round results Consent form topic (\% who
identified nothing important in
round $1 /$ round 2 )

Biobank purpose (8/0)

Blood draw (8/2)

Collection of information (2/0)

Duration of storage (14/6)

Access to biospecimens/data (8/6)

Recontact (10/4)

Large-scale data sharing (16/4)

Risks (6/4)

Confidentiality protections (33/28)

Genetic Information Nondiscrimination Act (37/32)

Certificate of confidentiality (45/68)

Potential benefits (10/6)

Costs and payments (commercialization) (24/19)

Return of results (6/2)

Discontinuing participation (14/4)

Questions or problems (27/28)
Interim statement of understanding (\% who endorsed element in round 1/round 2)

"The purpose of this project is to collect (47/64) and store (57/81) samples (78/100) and health information (49/74) for use in future (51/81) genetic (29/26) research (76/100)."

"You are going to draw a small amount (29/23) of blood (92/98) from my arm (24/26). From this blood, you will be able to get DNA (20/23)."

"You will ask me some basic information (57/79) about myself and my family history (31/17) and will contact me (57/83) no more than once a year (45/26) to update this information. You will also collect information from my medical records $(84 / 100)$ from time to time (33/17). These things will happen for as long as my sample is stored in the biobank (33/19)."

"My sample and information will be stored (65/89) and used for research (29/51) forever (63/94) unless I decide to stop taking part (61/94) or you close the biobank (22/17)."

"Many other researchers (45/36) from all over the world (22/21) may study (78/94) my samples and information. There will be a review of each request $(24 / 21)$. You will not give researchers information that could identify me (53/64) without my permission (24/17)."

"Researchers may want to contact (31/21) me for more information (35/21) or to ask me to participate in more research (51/96). If this happens, someone from the biobank will contact (59/96) me first to make sure it is OK (22/4). I won't be contacted about more than four studies a year (22/2), and for each one I can choose (20/6) whether or not to take part."

"Some of my information, including genetic (35/32) information, might be put into a database (49/79) to be shared $(27 / 36)$ with others outside the biobank $(22 / 21)$ for use in research. Information that could identify me (29/36) will not be put into a database, but there is still a small chance $(41 / 77)$ that someone could trace my information back to me (67/96). Researchers always have a duty to protect (22/21) my information."

"There are some risks associated with the blood draw (29/26). There is also a small chance (47/53) that someone could get access to information about me (51/96) and use it against me (39/49) in some way. Things researchers learn could also be used to support harmful ideas about groups (22/13) of people. You will do everything you can (29/19) to prevent my information from being used in harmful ways (20/11)."

"You will take many steps to protect my privacy (43/72), including not giving out information that could identify me (20/13)."

"There is a law (45/68) that protects me (33/38) from discrimination (51/68) by health insurers (37/38) and employers (35/38) based on my genetic (31/38) information. However, the law does not protect me (39/60) from genetic discrimination by life insurance (22/11) and long-term care insurance (20/11) companies."

"You have a certificate (20/28) that says that you do not have to give out my personal information (22/32). However, under some circumstances, you may still have to disclose (29/34) my information to certain authorities."

"I should not expect to benefit (90/94) directly (59/28) from this research. It may help other people (20/13)." "It will not cost (37/19) me anything to participate in the biobank. You will give me a $\$ 25$ gift card (35/26) for participating. Research done using my sample might someday lead to the development of a product (39/30) that could be sold. Although others may profit (31/6) from these products, I will not get any money (55/81) if this happens."

"I should not expect to get individual results (76/98) back from this research, unless you find something important to my health (63/49). If that happens, you will contact me (53/49) to see if I want to learn more (22/11). Research is not the same as medical care (29/11)."

"I have the right to leave (76/96) the project at any time (55/19). However, I cannot withdraw or get back (65/79) samples and information from studies that have already begun."

"There is a phone number (24/11) in the form I can call (67/72) if I have questions or want more information (63/72)."

Results are displayed using the most detailed statement presented in the round 2 survey, which incorporate elements endorsed by at least $20 \%$ of panelists in round 1 .

as enjoyable and thought provoking and the process as well organized and easy to follow. A few provided critiques, for example, about the length of the process, a preference for more qualitative input in round 3, and of Delphi techniques in general as "academic." One wondered whether focusing on one consent topic at a time may have led to an overestimate of what participants need to know. However, most panelists looked forward to the final results with anticipation that they would be useful to the field.

\section{DISCUSSION}

Valid informed consent requires that individuals understand and voluntarily agree to participate in research. ${ }^{23}$ Yet numerous studies have shown that participants in a variety of settingsincluding biobanking ${ }^{24-26}$-fail to grasp important aspects of the research to which they consented. Many commentators identify one source of the problem as equating informed consent solely with the requirement to disclose information. ${ }^{27-29}$ Disclosure is indeed a key aspect of informed consent, and 
Table 3 Final round results $(n=47)$

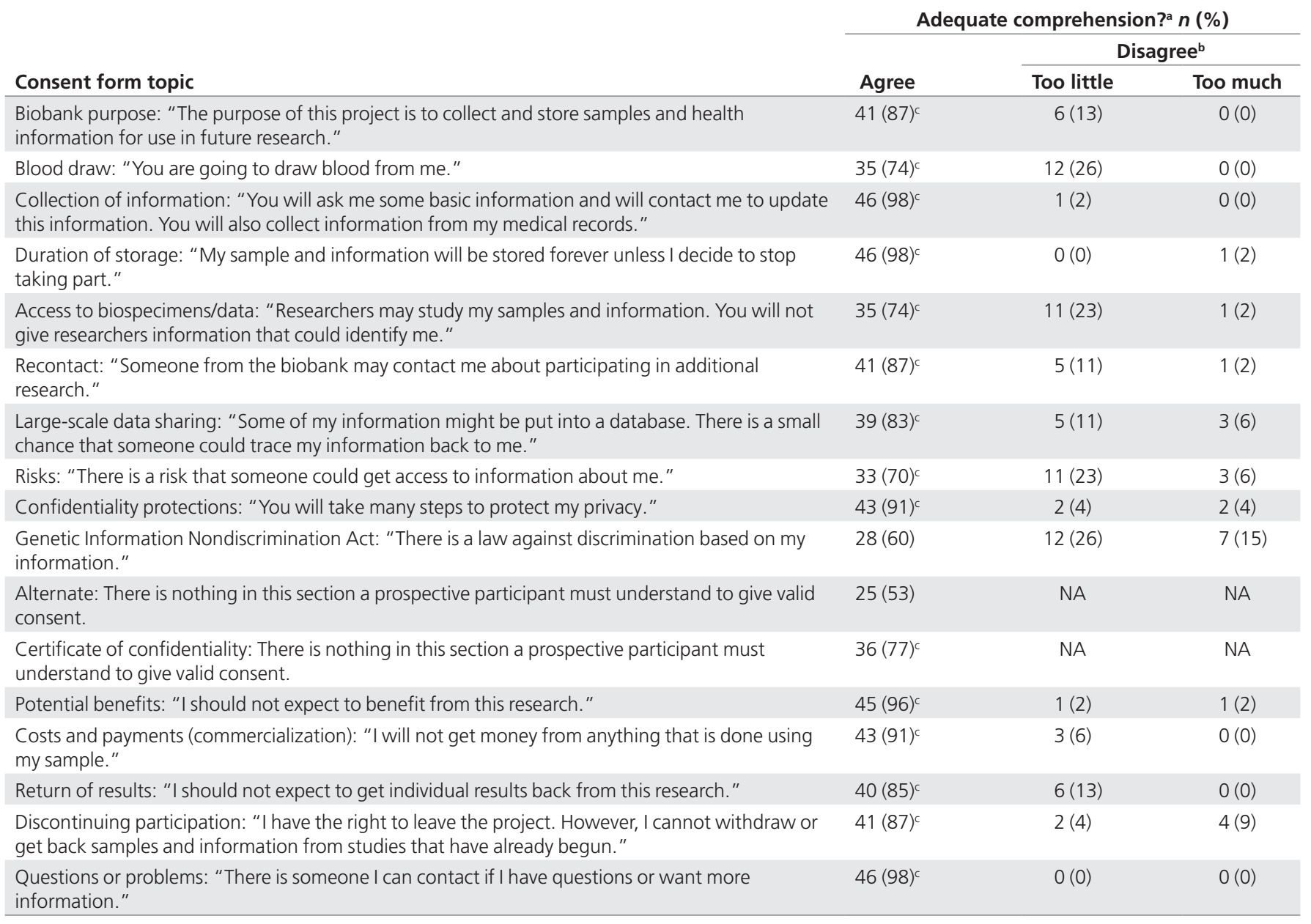

agreed or strongly agreed on a four-point scale in response to "How much do you agree or disagree that the statement above generally represents adequate comprehension of the consent topic?" bAmong those who disagreed or strongly disagreed that the statement represents adequate comprehension, responses to question about whether the statement reflects more or less than a prospective participant needs to understand to provide valid consent. 'Reached consensus, predefined as $\geq 70 \%$ agreement.

federal regulations and best practice guidelines provide lists of topics that should be covered in consent forms. Although these are useful starting points, they are not identical to the elements that individuals must understand to give valid consent. ${ }^{23}$ As stated by Appelbaum ${ }^{12}$ :

Consensus is needed on the question of what information must be understood and to what degree before subjects can be said to have adequate comprehension. . .. Research regulations generally elide this problem with lists of information to be disclosed as if all bits of data were equivalent. It is completely unrealistic, however, to expect research subjects to understand everything they are told, given the scope of most consent forms and the inherent complexity of projects, and some of what is communicated is clearly quite tangential to comprehension of the study itself. (p. 2)

Without consensus on which information is most crucial for participants to understand, it is difficult to operationalize researchers' ethical obligation to ascertain comprehension ${ }^{30}$ or to make progress on improving forms and processes to ensure valid consent. ${ }^{31}$

We attempted to meet this need in biobanking consent by engaging a diverse group of experts in a systematic process to define "adequate understanding." Our results reflect a high degree of consensus on a relatively small number of key points that prospective participants must grasp to provide valid consent. This outcome was achieved despite significant initial variability of opinion on the panel, which included genome scientists, biobank managers, ethics and policy experts, and community/participant representatives. Through a series of interrelated surveys in which panelists were able to consider results from prior rounds and perhaps think about informed consent in new ways (Table 4), there was clear movement toward consensus and ultimately agreement. It is not uncommon for empirical studies of ethical issues to reveal discordant views among stakeholders-including our own research on the content of biobank consent forms. ${ }^{32}$ Our present findings suggest there is reason for optimism that common ground can be reached upon further work and reflection. 
Table 4 Examples of panelists' comments on biobanking consent and Delphi process

Topic

Biobanking consent (GINA)

Delphi process

\section{Comment}

Skepticism about legislation: "Interesting result with respect to the GINA language-l do wonder if this is more a reflection of respondents' general skepticism about the legislation as a remedy for discrimination than an indication of preferences for comprehension?"

Need to know limitations: "With regard to [GINA], if it is important for participants to know that laws are in place to protect genetic information, then they would also need to know that there are limitations to these laws."

"I would prefer that this section be omitted entirely (i.e., no reference to GINA in the consent document); however, if it is included, then participants should be required to recognize that there is a law seeking to protect them from discrimination...but that it has limitations."

Gained new insights: "This survey (and the iterative process) has been fascinating and very informative. It has helped provide insight into the consent from the perspective of a participant."

"This has been very interesting. When looked at closely, there were a number of areas which seemed important, that upon reflection do not seem to impact a patient's comprehension. This has helped me view the consent process from a new perspective."

"It was interesting and enlightening. Really made me think about the whole purpose of informed consent and what subjects really need to know."

Well-organized, easy to follow: "An easy and interesting process."

"You provided the information in a clear and accessible way."

"I really enjoyed the Delphi process. The instructional videos at the beginning of each stage were put together well and provided good instructions."

Long process: "The process was quite long, and in places, tedious. It made me think about how participants feel about the informed consent process itself. I also realized all over again, how challenging many of the concepts are to convey to participants, and how challenging they are to understand, even for a person who regularly works with them."

Overestimate of knowledge needed for "adequate" comprehension: "I was pleasantly surprised to see that most people did not want tons of disclosure, but I still fear that the clause by clause approach leads to too much product."

Useful to the field: "This was fun. I feel as though progress is being made. I hope you rush preliminary results into publication, because IRBs and investigators are grappling with trying to streamline the biobank consent process right now."

"I look forward to the final analysis and recommendations to apply as a standard."

GINA, Genetic Information Nondiscrimination Act; IRB, institutional review board.

Table 5 Example of consent form "executive summary"

We would like to store some of your blood and health information in the Duke Biobank for use in research on health and disease. You can take part in this storage project or not. This consent form gives information to help you decide. Please read it carefully and take all the time you need to make your choice. Some of the most important things for you to know are:

- The purpose of this project is to collect and store samples and health information for use in future research.

- We will draw blood from you. We will ask you some basic information and will contact you to update this information. We will also collect information from your medical records.

- Your sample and information will be stored forever unless you decide to stop taking part.

- Researchers may study your samples and information. We will not give them information that directly identifies you.

- Someone from the biobank may contact you about participating in additional research.

- Some of your information might be put into a database. There is a small chance that someone could trace your information back to you.

- We will take many steps to protect your privacy, but there is a risk that someone could get access to information about you. There is a law against discrimination based on your information.

- You should not expect to benefit from this research. You will not get money from anything that is done using your sample. You should not expect to get individual results back from this research.

- You have the right to leave the project. However, we cannot withdraw or get back samples and information from studies that have already begun.

- There is someone you can contact if you have questions or want more information.

Readability characteristics: Flesch-Kincaid grade level 7.3; Flesch-Kincaid reading ease 65.2 (test rates text on 100-point scale; the higher the score, the easier it is to understand); passive sentences $9 \%$.

GINA was the only consent topic for which consensus was not achieved. Opinions were especially varied in early rounds, leading to this being the only topic for which two statements were presented for consideration in the final survey. It is possible that the lack of consensus for GINA in the final round was in part because of having two options to evaluate rather than one. Pending further research, whether knowledge of GINA is essential for valid consent will be a matter of investigator judgment.

Interpretation of our results is subject to several limitations. First, our panel lacked cultural diversity. Subject matter expertise was the primary inclusion criterion, and we identified panelists based on membership in existing committees and consortia; thus the cultural diversity of the panel reflects that 
of these underlying entities. Second, we asked panelists about existing model consent language for a general, non-diseasespecific biobank such as might be established at any academic medical institution. Biobanks that focus on a particular disease or that are established within special populations ${ }^{5}$ may require different levels or types of understanding for valid consent. In general, investigators looking to assess comprehension may choose to tailor our results to fit their study context; interim findings from our earlier rounds (Table 2) suggest candidates for additional elements of information that some may deem essential. Third, our study was conducted in the United States, with panelists and consent language based on the US context. Although further work is needed, our results may still be useful in other settings because approaches to biobanking are not only similar around the world but are being harmonized to enable global data sharing. ${ }^{33}$

The goal of our study was not to identify which information should be disclosed to prospective biobank participants, but rather which details among those disclosed are essential for participants to understand. We believe our results may be useful in three ways:

(i) Improve consent forms: Consent forms must meet federal regulatory requirements; in addition, they should be responsive to best practice guidelines and focus on information most important to prospective participants. ${ }^{32,34}$ Within these parameters, our results may be helpful in organizing and formatting forms to highlight the most essential information; less important details may be candidates for removal when not needed for another reason or moved to supplemental material. Items listed in Table 3 could also serve as an introductory "executive summary" (Table 5) to a consent form or provide the basis for a brief, standard form, as has been advocated elsewhere. ${ }^{35,36}$

(ii) Improve consent processes: Similarly, consent processeswhich will increasingly be undertaken in a variety of scalable ways-could be directed to focus on effectively communicating the most crucial information. Items in Table 3 could serve as the basis for developing tools and assessing comprehension in practice (e.g., through a teach-back tool or online quiz). ${ }^{37,38}$

(iii) Facilitate research on biobanking consent: Lack of common definitions and standardized tools for assessing understanding have led to "conceptual and methodologic chaos"12 in studies of research participant understanding ${ }^{31}$ and interventions to improve informed consent. ${ }^{38}$ Our results help remedy this for biobanking; items in Table 3 provide an absolute metric of adequate understanding-an improvement over relative assessments of which intervention produces better scores than another without knowing whether either is adequate.

Next steps in our own program of research include using these results to develop practical ways to assess comprehension and to use these tools to determine the effect of a simplified biobanking consent form on participant comprehension. Further areas for future research include potentially using similar steps and methodologies to improve informed consent in other contexts (e.g., phase III clinical trials). Delphi techniques explicitly make use of expert opinion, that is, they involve individuals with substantial existing knowledge of the topic area. Additional research could make use of deliberative democracy methods ${ }^{39-41}$ to elicit informed and wellconsidered opinions about consent comprehension among the general public.

\section{SUPPLEMENTARY MATERIAL}

Supplementary material is linked to the online version of the paper at http://www.nature.com/gim

\section{ACKNOWLEDGMENTS}

We thank the following individuals, who provided input as members of the advisory group assembled for this grant: John Alexander, MD, MHS (Duke University); Jeffrey R. Botkin, MD, MPH (University of Utah); John M. Falletta, MD (Duke University); Steven Joffe, MD, MPH (University of Pennsylvania); Bartha Maria Knoppers, PhD (McGill University); Karen J. Maschke, PhD (The Hastings Center); P. Pearl O'Rourke, MD (Partners Healthcare). We also thank Li Lin, MS (Duke University), for her assistance in preparing the figure in Supplementary Appendix S3 online. Those named here were compensated for their roles on the project. This work was supported by a grant (R01HG006621) from the National Human Genome Research Institute (NHGRI). The content is solely the responsibility of the authors and does not necessarily represent the official views of the NHGRI or the National Institutes of Health. The funders had no role in study design, data collection and analysis, decision to publish, or preparation of the manuscript.

\section{DISCLOSURE}

The authors declare no conflict of interest.

\section{REFERENCES}

1. Vaught JB, Henderson MK, Compton CC. Biospecimens and biorepositories: from afterthought to science. Cancer Epidemiol Biomarkers Prev 2012;21:253255.

2. Henderson GE, Cadigan RJ, Edwards TP, et al. Characterizing biobank organizations in the U.S.: results from a national survey. Genome Med 2013;5:3

3. Baker M. Biorepositories: building better biobanks. Nature 2012;486:141-146.

4. Emanuel EJ, Menikoff J. Reforming the regulations governing research with human subjects. N Engl J Med 2011;365:1145-1150.

5. Mello MM, Wolf LE. The Havasupai Indian tribe case-lessons for research involving stored biologic samples. N Engl J Med 2010;363:204-207.

6. Couzin-Frankel J. Ethics. DNA returned to tribe, raising questions about consent. Science 2010;328:558.

7. Doerr A. Newborn blood spot litigation: 70 days to destroy 5+ million samples. http://www.genomicslawreport.com/index.php/2010/02/02/newborn-bloodspot-litigation-70-days-to-destroy-5-million-samples/. Accessed 17 June 2014.

8. Callaway E. HeLa publication brews bioethical storm. http://www.nature.com/ news/hela-publication-brews-bioethical-storm-1.12689. Accessed 17 June 2014.

9. Vaught J, Lockhart NC. The evolution of biobanking best practices. Clin Chim Acta 2012:413:1569-1575.

10. McGuire AL, Beskow LM. Informed consent in genomics and genetic research Annu Rev Genomics Hum Genet 2010;11:361-381.

11. Wendler D, Grady C. What should research participants understand to understand they are participants in research? Bioethics 2008;22:203-208.

12. Appelbaum PS. Understanding "understanding": an important step toward improving informed consent to research. AJOB Prim Res 2010;1(suppl 2): $1-3$. 
13. Fink A, Kosecoff J, Chassin M, Brook RH. Consensus methods: characteristics and guidelines for use. Am J Public Health 1984;74:979-983.

14. Black N, Murphy M, Lamping D, et al. Consensus development methods: a review of best practice in creating clinical guidelines. $J$ Health Serv Res Policy 1999:4:236-248.

15. Hasson F, Keeney S, McKenna H. Research guidelines for the Delphi survey technique. J Adv Nurs 2000;32:1008-1015.

16. Hutchings A, Raine R, Sanderson C, Black N. A comparison of formal consensus methods used for developing clinical guidelines. J Health Serv Res Policy 2006;11:218-224.

17. Keeney S, Hasson F, McKenna HP. The Delphi technique in nursing and health research. Wiley-Blackwell: Chichester, West Sussex, UK, 2011.

18. Graham B, Regehr G, Wright JG. Delphi as a method to establish consensus for diagnostic criteria. J Clin Epidemio/ 2003;56:1150-1156.

19. The Electronic Medical Records and Genomics (eMERGE) Network Consent \& Community Consultation Workgroup Informed Consent Task Force. Model consent language. http://www.genome.gov/27526660. Accessed 17 June 2014.

20. U.S. Department of Health \& Human Services. Office for Human Research Protections. Guidance on the Genetic Information Nondiscrimination Act: Implications for Investigators and Institutional Review Boards. http://www.hhs. gov/ohrp/policy/gina.html. Accessed 17 June 2014.

21. Beskow LM, Check DK, Ammarell N. Research Participants' Understanding of and Reactions to Certificates of Confidentiality. AJOB Prim Res 2014;5:12-22.

22. Hudson KL, Holohan MK, Collins FS. Keeping pace with the times-the Genetic Information Nondiscrimination Act of 2008. N Engl J Med 2008;358:26612663.

23. Wendler D. Can we ensure that all research subjects give valid consent? Arch Intern Med 2004;164:2201-2204.

24. McCarty CA, Nair A, Austin DM, Giampietro PF. Informed consent and subject motivation to participate in a large, population-based genomics study: the Marshfield Clinic Personalized Medicine Research Project. Community Genet 2007; 10:2-9

25. Ormond KE, Cirino AL, Helenowski IB, Chisholm RL, Wolf WA. Assessing the understanding of biobank participants. Am J Med Genet A 2009;149A:188198.

26. Rahm AK, Wrenn M, Carroll NM, Feigelson HS. Biobanking for research: a survey of patient population attitudes and understanding. J Community Genet 2013;4:445-450

27. Beauchamp TL, Childress JF. Principles of Biomedical Ethics. 4th Ed. Oxford University Press: New York; 1994.
28. National Bioethics Advisory Commission. Ethical and Policy Issues in Research Involving Human Participants, Volume 1. US Government Printing Office: Rockville, MD, 2001.

29. Institute of Medicine. Responsible Research: A Systems Approach to Protecting Research Participants. National Academies Press: Washington, DC, 2002.

30. National Commission for the Protection of Human Subjects of Biomedical and Behavioral Research. The Belmont Report: Ethical Principles and Guidelines for the Protection of Human Subjects of Research. US Government Printing Office: Washington, DC, 1979.

31. Sand K, Kaasa S, Loge JH. The understanding of informed consent information-definitions and measurements in empirical studies. AJOB Prim Res 2010;1(suppl 2):4-24.

32. Beskow LM, Friedman JY, Hardy NC, Lin L, Weinfurt KP. Simplifying informed consent for biorepositories: stakeholder perspectives. Genet Med 2010;12:567-572.

33. White Paper. Creating a Global Alliance to Enable Responsible Sharing of Genomic and Clinical Data. http://www.broadinstitute.org/files/news/pdfs/ GAWhitePaperJune3.pdf. Accessed 17 June 2014.

34. Beskow LM, Friedman JY, Hardy NC, Lin L, Weinfurt KP. Developing a simplified consent form for biobanking. PLOS One 2010;5:e13302.

35. National Cancer Institute. Office of Biorepositories and Biospecimen Research. Custodianship and Ownership Issues in Biospecimen Research. http:// biospecimens.cancer.gov/global/pdfs/CaOSumm.pdf. Accessed 17 June 2014.

36. Department of Health and Human Services. Advance Notice of Proposed Rulemaking: Human Subjects Research Protections-Enhancing Protections for Research Subjects and Reducing Burden, Delay, and Ambiguity for Investigators. Federal Register 2011;76(suppl 143):44512-44531.

37. Palmer BW, Cassidy EL, Dunn LB, Spira AP, Sheikh Jl. Effective use of consent forms and interactive questions in the consent process. IRB 2008;30:8-12.

38. Flory J, Emanuel E. Interventions to improve research participants understanding in informed consent for research: a systematic review. JAMA 2004;292:1593-1601.

39. De Vries R, Stanczyk A, Wall IF, Uhlmann R, Damschroder L, Kim SY. Assessing the quality of democratic deliberation: a case study of public deliberation on the ethics of surrogate consent for research. Soc Sci Med 2010;70:1896-1903.

40. Kim SY, Wall IF, Stanczyk A, De Vries R. Assessing the public's views in research ethics controversies: deliberative democracy and bioethics as natural allies. $J$ Empir Res Hum Res Ethics 2009;4:3-16.

41. Secko DM, Preto N, Niemeyer S, Burgess MM. Informed consent in biobank research: a deliberative approach to the debate. Soc Sci Med 2009;68:781789. 